\title{
Southampton
}




\section{eXtensible Stylesheet Language}

\section{COMP3220 Web Infrastructure}

Dr Nicholas Gibbins - nmg@ecs.soton.ac.uk 


\section{The Problem}

How do you maintain different versions of a document for presentation on different systems that:

- Have different presentation characteristics (screen size, etc)?

- Require different document formats?

XSL is a family of XML-based technologies designed to address this problem 


\section{eXtensible Stylesheet Language}

XSL Transformations (XSLT)

- XML-based language for describing transformations from one XML-based language to another

XML Path Language (XPath)

- Language for referring to parts of an XML document

XSL Formatting Objects (XSL-FO)

- XML vocabulary for specifying formatting semantics 


\section{XML processing}

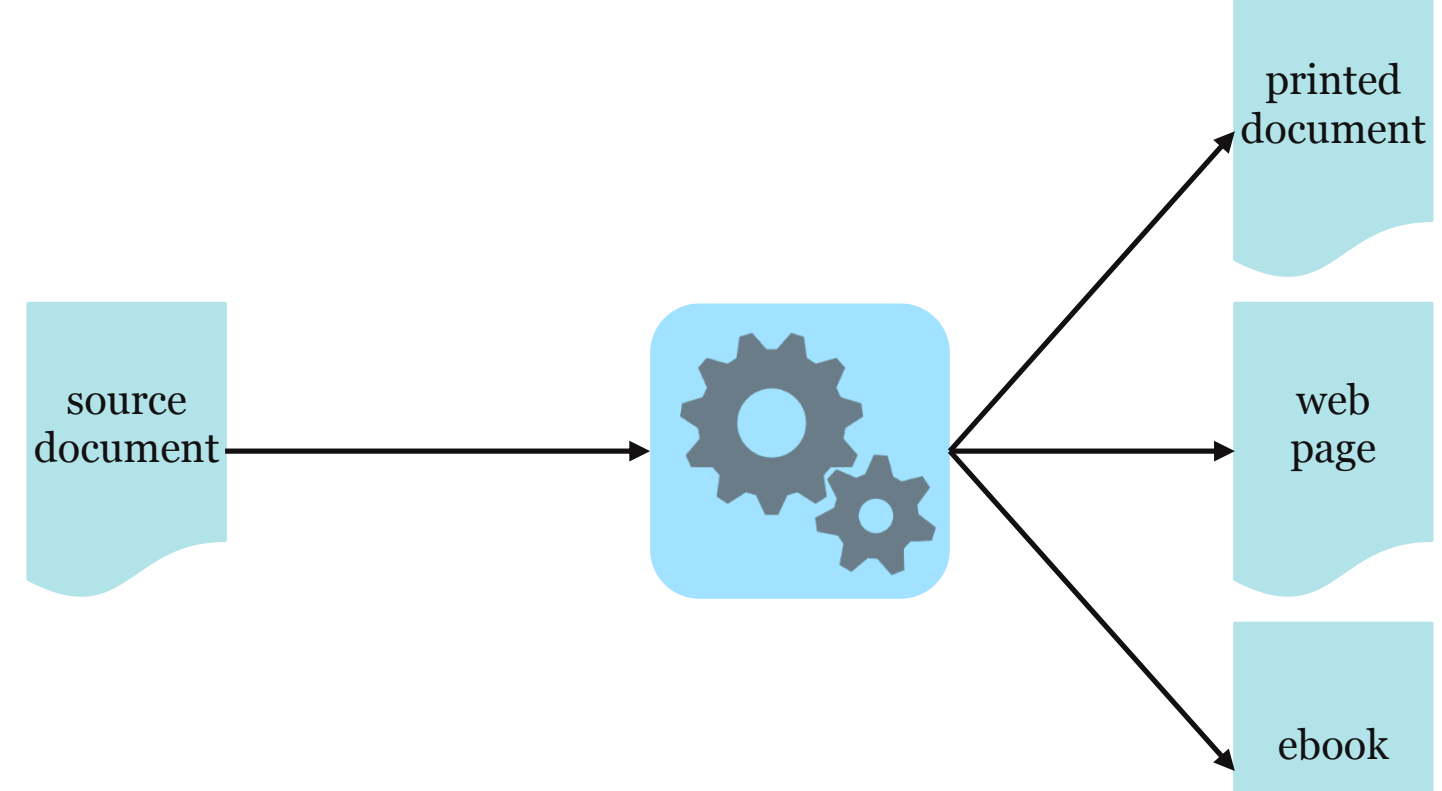




\section{XSL processing in theory}

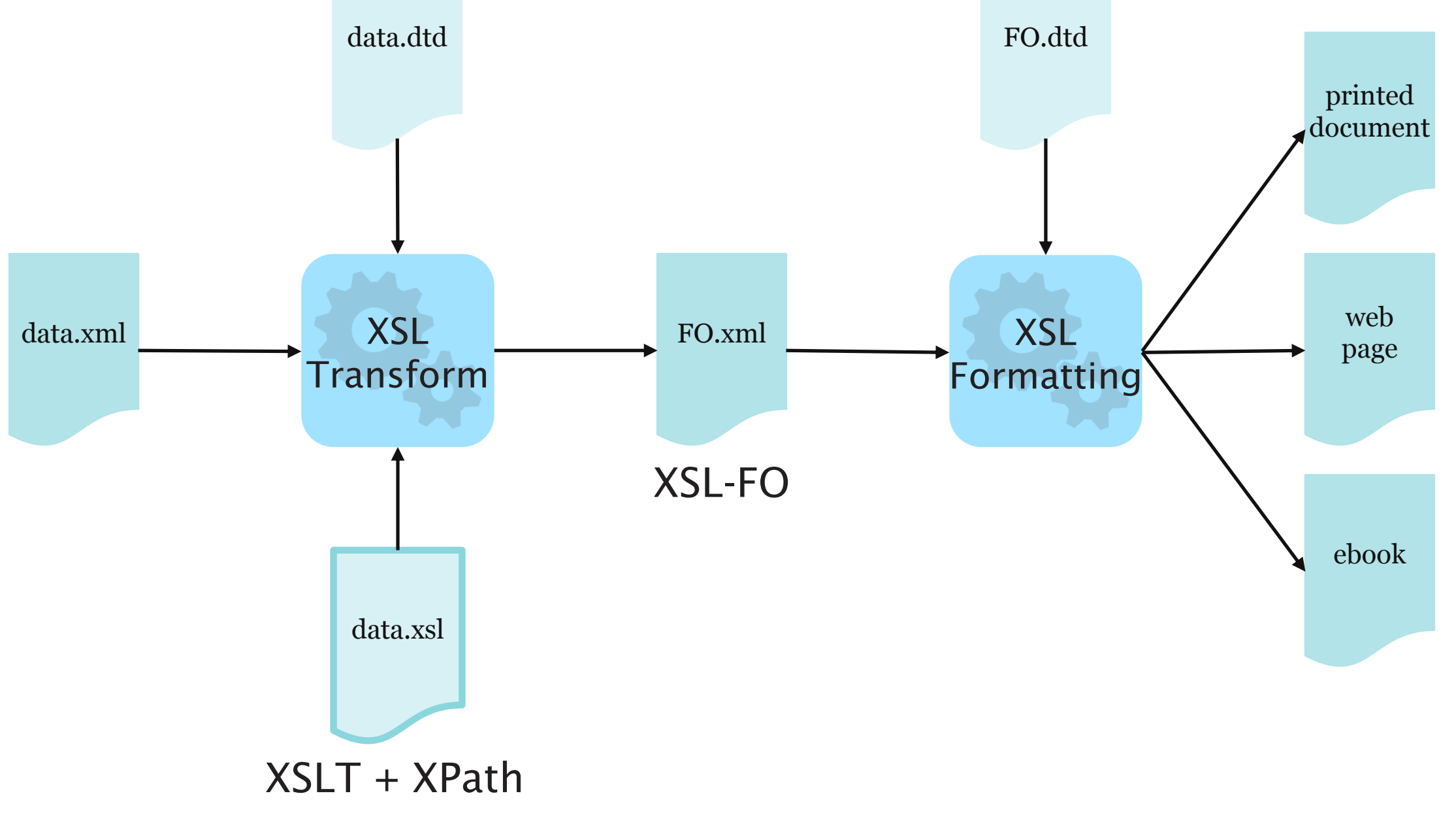




\section{XSL processing in practice}

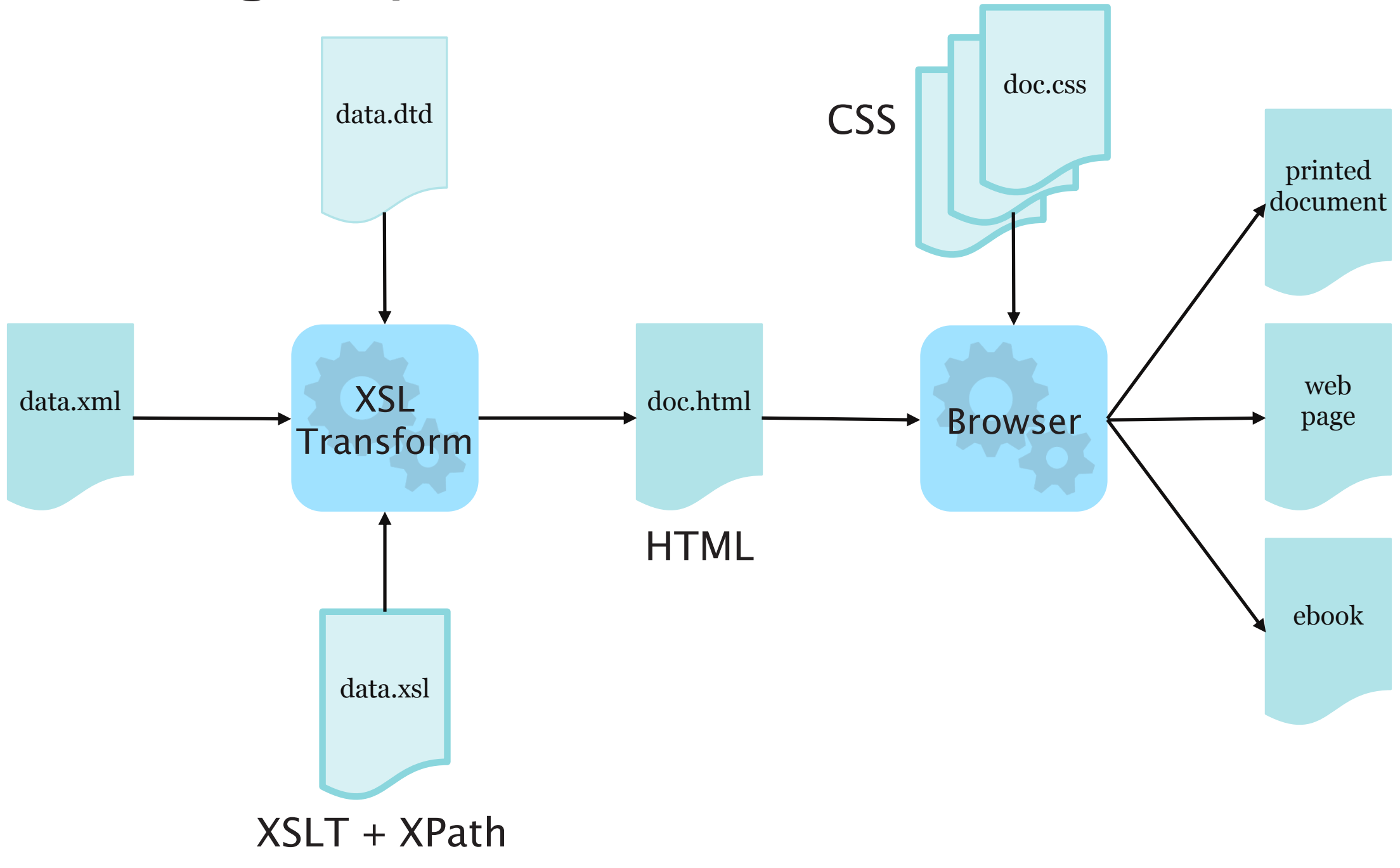




\section{Example input}

$<$ ?xm7 version="1.0" encoding="UTF-8"?> $<$ contacts>

$<$ name $>$ My address 1ist $</$ name $>$

$<$ card $>$

$<$ name $>$ Fred Foo</name $>$

$<$ emai 1>fred@examp1e.org</emai 1>

$<$ te1 type="work">01234567890</te1>

$</$ card $>$

$<$ card $>$

$<$ name $>$ Ji11 Bar $<$ /name $>$

<emai1>ji11@examp1e.org</emai1>

$<$ te1 type="home">02345678901</tel>

$</$ card $>$

$</$ contacts $>$ 


\section{Example output}

<htm1 xm1ns="http://www.w3.org/TR/xhtm11/strict"> $<$ head $>$

$<$ title $>$ My address 1ist $</$ title $>$

$</$ head $>$

$<$ body $>$

$<$ h1 $>$ My address 1ist $</$ h1 $>$

$<$ div $>$

$<$ h2 $>$ Fred Foo $</$ h $2>$

$<$ p Emai1:

$<$ a href="mai1 to: fred@example.org" $>$ fred@example.org $</ a></ p>$ $</$ div $>$

$<$ div $>$

$<$ h2>Ji11 Bar $</$ h2 $>$

$<$ p Emai1:

$<$ a href="mai1to:ji11@examp1e.org" $>$ ji11@examp1e.org $</ a></ p>$ $</$ body $>$

$<$ p $>$ Telephone: $02345678901</ p></$ div $>$

$</$ html $>$ 


\section{XSL stylesheets}

An XSL stylesheet consists of a number of templates

\section{Each template:}

- Matches an element in the original document using XPath

- Specifies the new content with which the element is to be replaced 


\section{XPath}

Expression language for specifying nodes (elements, attributes) within an XML document

Specifies nodes using a path through the hierarchy of the document

- Can be absolute (from the root) or relative (from current position) 


\section{XPath expressions}

$E$

/

//

$\cdot$

$\cdots$

Q
Selects all nodes with the name $\mathrm{E}$

Selects from the root node

Selects nodes anywhere under the current node

Selects the current node

Selects the parent of the current node

Selects attributes 


\section{XPath expression examples}

contacts

/contacts

contacts/card

//card

contacts//name

//te1/@1ang
Selects all contacts elements

Selects the root contacts element

Selects all card elements that are children of contacts

Selects all card elements

Selects all name elements that are descendants of contacts

Selects type attribute on all te1 elements 


\section{xs1 : temp1ate}

match attribute contains XPath expression that identifies an element or elements

- Content of element is either output markup, or other XSL directives

<xs 1: template match="/">

$</ x s 1:$ temp1ate $>$ 


\section{xs1: app1y-temp1ates}

Used within body of a template

- Recursively applies templates to children of the element

- select attribute identifies target child node using XPath

<xs1:app1y-temp1ates select="..."/> 


\section{xs1: for-each}

Loops over every matching element

- select attribute identifies target node set

<xs1: for-each select="...">

$</ x s 1:$ for-each $>$ 


\section{xs1:value-of}

Extracts the value of an XML element and uses it in the output document

- select attribute used to identify element

<xs1:value-of select="..."/> 


\section{XSLT example - input}

$<$ ?xm7 version="1.0" encoding="UTF-8"?> $<$ contacts $>$

$<$ name $>$ My address 1ist $</$ name $>$

$<$ card $>$

$<$ name $>$ Fred Foo</name $>$

<emai 1>fred@examp1e.org</emai 1>

$<$ te1 type="work">01234567890</te1>

$</$ card $>$

$<$ card $>$

$<$ name $>$ Ji11 Bar $<$ /name $>$

<emai1>ji11@examp1e.org</emai1>

$<$ te1 type="home">02345678901</tel>

$</$ card $>$

$</$ contacts $>$ 


\section{XSLT example - output}

<htm1 xm1ns="http://www.w3.org/TR/xhtm11/strict"> $<$ head $>$

$<$ title $>$ My address 1ist $</$ title $>$

$</$ head $>$

$<$ body $>$

$<$ h1 $>$ My address 1ist $</$ h1 $>$

$<$ div $>$

$<$ h2 $>$ Fred Foo $</$ h2 $>$

$<$ p Email :

$<$ a href="mai1 to: fred@example.org" $>$ fred@example.org $</ a></ p>$ $</$ div $>$

$<$ div $>$

$<$ h2>Ji11 Bar $</$ h2 $>$

$<$ p Emai1 :

$<$ a href="mai1to:ji11@example.org">ji11@example.org $</ a></ p>$ $</$ body $>$

$<$ p $>$ Telephone: $02345678901</ p></$ div $>$

$</$ html $>$ 


\section{XSLT example - using stylesheet}

$<$ ?xm1 version="1.0" encoding="UTF-8"?>

$<$ ?xm1-stylesheet type="text/xs1" href="contacts.xs1" version="1.0"?>

$<$ contacts>

<name $>$ My address 1ist $</$ name $>$

$<$ card $>$

$<$ name $>$ Fred Foo $<$ /name $>$

<emai 1>fred@examp1e.org</emai 1>

$<$ te $1>01234567890</$ tel $>$

$</$ card $>$

$<$ card $>$

$<$ name $>$ Ji11 Bar</name>

<emai 1>ji11@examp1e.org</emai 1>

$<$ tel>02345678901</tel>

$</$ card $>$

$</$ contacts $>$ 


\section{XSLT example - stylesheet}

$<$ ?xm1 version="1.0" encoding="UTF-8"?>

$<x s 1$ : stylesheet version="1.0"

xm1ns:xs1="http://www.w3.org/1999/XSL/Transform"

xm7ns="http: //www.w3.org/TR/xhtm11/strict">

$</ x$ s1: stylesheet> 


\section{XSLT example - templates}

$<x$ 1: template match="te1">

$<$ p>Telephone: <xs1:value-of select="."/></p>

$</ x$ s 1 : template $>$ 


\section{XSLT example - templates}

$<x$ 1: template match="emai1">

$<$ p $>$ Emai1: <a href="mailto: $\{\} ">.<x s 1:$ value-of select="." $/></ a></ p>$ $</ x s 1:$ template $>$ 


\section{XSLT example - templates}

$<$ xs1: template match="name" $>$

$<$ h2 $<<$ xs 1:value-of select="."/></h2>

$</ x \mathrm{x} 7$ : template $>$ 


\section{XSLT example - templates}

<xs1: temp1ate match="/">

$<$ html $>$

$<$ head $>$

$<$ title $><$ xs1:value-of select="/contacts/name"/></title $>$ $</$ head $>$

$<$ body $>$

$<$ h1 $><x$ 1: value-of select="/contacts/name"/ $></$ h1 $>$

$<$ xs1:for-each select="/contacts/card">

$<$ div $>$

<xs1:app1y-temp1ates select="name"/>

<xs1:apply-templates select="emai1"/>

$<$ xs1:app1y-templates select="te1"/>

$</$ div $>$

$</$ xs 1: for-each>

$</$ body $>$

$</$ html $>$

$</ x s 1$ : template $>$ 


\section{XSL versus CSS}

Considering CSS...

Pros

- Simple (relatively)

- Cascading recognises different needs of users and authors

Cons

- Unable to modify document structure 


\section{XSL versus CSS}

Considering XSL:

Pros

- Able to modify and transform document structure

- Better for data

Cons

- Complex (relatively)

- Cumbersome for ordinary documents

- No consideration of differing needs of users versus authors 


\section{Next Lecture: Optimising HTTP}

